\title{
Familiares cuidadores de usuários de serviço de saúde mental: Satisfação com serviço
}

\author{
Ana Flávia de Oliveira Santos \\ Cármen Lúcia Cardoso \\ Universidade de São Paulo
}

\begin{abstract}
Resumo
Com a reforma psiquiátrica, os familiares foram chamados a atuar como parceiros no cuidado aos usuários. O papel desempenhado como articuladores da relação serviço-usuário conferiu importância a sua participação na avaliação da qualidade da assistência. O estudo objetivou avaliar a satisfação com o serviço de 54 familiares cuidadores de usuários de serviço substitutivo em saúde mental. Utilizaram-se os instrumentos: Escala de Avaliação da Satisfação dos Familiares em Serviços de Saúde Mental (SATIS-Br) e questionário sociodemográfico. Os dados foram tratados através de estatística descritiva e estatística não-paramétrica. Os cuidadores apresentaram satisfação de moderada a alta. Verificou-se correlação negativa entre satisfação quanto aos resultados do tratamento e a variável idade do usuário $(r$ $=-0,37 ; p=0,006)$. Os cuidadores apontaram principalmente a ampliação de atividades no serviço como estratégia de melhora da assistência. Concluiu-se que os serviços substitutivos devem avançar na proposta da intersetorialidade e otimizar recursos próximos à localidade das famílias.
\end{abstract}

Palavras-chave: avaliação de serviços de saúde; cuidadores; saúde mental.

\begin{abstract}
Family caregivers of mental health service users: Satisfaction with the service. Given the psychiatric reform, family members of mental patients were invited to act as partners in the care provided to users. The role these individuals perform in the relationship service-user conferred importance to their evaluation of quality of care. This study investigates the satisfaction of 54 family caregivers of users of substitute mental health services. The following were used: The Brazilian Mental Health Services' Family Satisfaction Scale (SATIS-BR) and a socio-demographic questionnaire. Data were treated through descriptive and non-parametric statistics. Caregivers presented moderate to high satisfaction. A negative correlation was observed between 'satisfaction with the treatment results' and the variable 'age of users' ( $p=$ 0.006). The caregivers mainly indicated broadening activities as a strategy to improve care. The conclusion is that the substitute services should advance in terms of inter-sector cooperation and optimize resources close to the residence of families.
\end{abstract}

Keywords: health services evaluation; caregivers; mental health.

\section{Resumen}

Familiares cuidadores de usuarios del servicio de salud mental: Satisfacción con el servicio. Con la reforma psiquiátrica, los familiares fueron invitados a actuar como compañeros en el cuidado a los usuarios. El papel desempeñado como articuladores de la relación servicio-usuario le dio importancia a su participación en la evaluación de la calidad de la asistencia. El estudio tuvo como objetivo evaluar la satisfacción con el servicio de 54 familiares cuidadores de usuarios de servicio sustitutivo en salud mental. Se utilizaron los instrumentos: Escala de Avaliação da Satisfação dos Familiares em Serviços de Saúde Mental (SATIS-Br) y cuestionario sociodemográfico. Los cuidadores presentaron satisfacción de moderada a alta. Se verificó correlación negativa entre satisfacción de los resultados del tratamiento y edad del usuario. Los cuidadores señalaron la ampliación de actividades en el servicio como estrategia de mejora de la asistencia. Se concluyó que los servicios sustitutivos deben avanzar en la propuesta de la intersectorialidad y optimizar recursos cercanos a la localidad de las familias.

Palabras clave: evaluación de servicios de salud; cuidadores; salud mental. 
A avaliação de serviços de saúde tem por objetivo avaliar a qualidade da assistência e do cuidado, que, conforme assinala Donabedian (1988), deve ser considerada sob o tripé estrutura, processo e resultado, que reúne uma proposta de avaliação global dos serviços. A estrutura compreende os recursos materiais e humanos, bem como a própria estrutura organizacional. O processo abarca o manejo dos problemas apresentados pelo usuário; já o resultado significa o efeito dos cuidados no estado de saúde dos usuários.

No contexto da saúde mental, a avaliação do cuidado vem ganhando destaque. A Organização Mundial da Saúde - OMS (2001) propôs, dentre as recomendações para a área, a realização de pesquisa e monitoramento dos serviços, que fornecem indicadores de saúde mental tais como adequação da assistência, qualidade das intervenções preventivas, avaliação dos resultados do tratamento, entre outros.

Para Vasconcelos (1995), as iniciativas de avaliação sistemática de políticas e programas sociais e de saúde no Brasil ainda são escassas, o que indica a necessidade de um processo de avaliação rigorosa dos modelos clínicos e institucionais no contexto da Reforma Psiquiátrica, visando verificar sua efetividade, bem como fornecer parâmetros normativos para estas novas instituições e seus gestores. Pitta (1996) destaca a importância de se incorporar o processo de investigação como algo próprio do desenvolvimento dos serviços, de modo contínuo e sistemático. Ao se adotar este ponto de vista, permite-se avaliar a qualidade da assistência, possibilitando constantes reavaliações do atendimento prestado e de seu impacto.

Uma medida de avaliação dos serviços cada vez mais utilizada diz respeito à satisfação das pessoas diretamente envolvidas no processo de cuidado, a saber: profissionais, usuários e familiares. Segundo Bandeira, Pitta e Mercier (2000), a avaliação da satisfação situa-se enquanto um dos indicadores de resultado de tratamento, sendo uma de suas principais referências da qualidade do serviço. Conforme pontuam Esperidião e Trad (2005), satisfação diz respeito à percepção subjetiva do indivíduo quanto ao atendimento que recebe nos serviços de saúde. Relaciona-se com a percepção de que o serviço atende às necessidades, indicando se as expectativas quanto ao tratamento foram - ou não - atingidas.

Dada a relevância do papel do familiar, tendo em vista a reforma na assistência em saúde mental, assim como sua participação cada vez mais ativa no cuidado ao usuário, torna-se central considerar sua perspectiva dentro da avaliação do cuidado. Segundo aponta Bandeira, Perreault, Mercier, Libério e Pitta (2002), a família é fonte primária de suporte aos usuários, possuindo a condição de detectar precocemente os resultados do tratamento, as dificuldades e as necessidades deles.

A percepção do familiar se mostra relevante dentro da perspectiva de maior participação social nos processos decisórios, podendo oferecer informações pertinentes tanto para o planejamento quanto para a avaliação da assistência dos serviços de saúde mental. Pode ainda contribuir para a elaboração de novas propostas de intervenção e readequação no funcionamento dos serviços, bem como oferecer auxílio aos gestores na elaboração de projetos e implementação das ações e programas de saúde, buscando apontar novos direcionamentos no cuidado e assegurar a qualidade da assistência.

Perreault et al. (2012) enfatizaram a necessidade de maior participação dos familiares tanto no tratamento quanto na avaliação dos serviços. Segundo Pegoraro e Caldana (2006), a compreensão da perspectiva dos familiares pode auxiliar a equipe na articulação de estratégias de trabalho voltadas ao usuário, bem como os próprios familiares. Para Kantorski et al. (2012), dados referentes à avaliação realizada por familiares permitem uma reorganização dos serviços no sentido de readequação da assistência às suas necessidades. Bandeira, Silva, Camilo e Felício (2011) enfatizam a insuficiência de estudos que contemplem a perspectiva do familiar na avaliação dos serviços de saúde mental.

Almeida (2002) verificou, em relação à satisfação com um serviço substitutivo em saúde mental do Rio de Janeiro, uma maior satisfação dos usuários, seguido pelos familiares e, por fim, pelos profissionais. Pereira e Cais (2001), em um estudo voltado à identificação do modo como a família percebe o serviço de saúde mental, verificaram visão associada a espaço de tratamento e convívio, além do acolhimento como necessidade de apoio ao enfrentamento dos conflitos vivenciados. A eficácia e eficiência do tratamento dependeriam, pois, dos vínculos formados no processo de assistência. Mello e Furegato (2008), em uma pesquisa de investigação sobre as representações de familiares acerca do Centro de Atenção Psicossocial (CAPS), verificaram identificação do serviço como trazendo alívio da sobrecarga gerada pela convivência prolongada com a pessoa com transtorno mental.

Em estudo desenvolvido por Bandeira et al. (2002), identificaram-se como pontos positivos dos serviços avaliados: atenção dos profissionais, competência com que exerciam seu trabalho e acolhimento aos usuários. Como necessidades de melhoria, apontaram: aumento de vagas para usuários novos; reforma e ampliação do espaço físico; aumento do número de atividades no serviço, com realização de atividade física; fornecimento de vale-transporte aos usuários; prolongamento do tempo de permanência do usuário no serviço; maior participação do familiar no tratamento; oferecimento de oficinas profissionalizantes; fornecimento de alimentação ao usuário; maior esforço da equipe para convencer o usuário a participar ainda mais do serviço.

Kantorski et al. (2009) verificaram avaliação positiva dos familiares quanto à qualidade do atendimento, diversidade de atividades, cuidado, vínculo e responsabilização. Schneider, Camatta, Nasi, Adamoli e Kantorski (2009), realizando avaliação de um serviço de saúde mental no Sul do país, verificaram que os familiares apresentaram satisfação com o ambiente físico e com as oficinas terapêuticas; ressaltaram a importância da visita domiciliar e da maior inserção do familiar no tratamento; reivindicaram maior número de profissionais. No estudo de Costa, Bandeira, Cavalcanti e Scalon (2011), verificouse predominância de familiares considerando beneficamente o tratamento dos usuários, ajudando-os a se sentir melhor, 
principalmente porque os ajudou a ficarem mais tranquilos, além do que foram valorizadas as atividades desenvolvidas pelos usuários.

Kantorski et al. (2012) identificaram alguns aspectos de satisfação entre os familiares de usuários de um CAPS, tais como no aspecto da ambiência do serviço, advinda da percepção de uma estrutura mais adequada; atendimento e acolhimento recebidos; oferecimento de oficinas que inserem os familiares no serviço e visita domiciliar. Aspectos ligados à insatisfação estiveram ligados a profissionais em número insuficiente. Perreault et al. (2012) verificaram a relação entre profissionais e familiares como mais fortemente atrelada ao grau de satisfação dos familiares.

Segundo Moreno e Alencastre (2004), a Reforma Psiquiátrica possibilitou a construção de uma rede de relações no sentido de estabelecer uma convivência saudável entre usuários, familiares, profissionais e a sociedade. Neste contexto, o processo de avaliação se mostra fundamental nos novos direcionamentos dos serviços e na verificação da efetividade das práticas a que se propõem.

Para Duarte (2007), apesar da importância dos estudos que priorizam a avaliação dos serviços substitutivos em saúde mental, estes ainda são escassos, tanto em relação à avaliação da qualidade desses serviços, quanto ao acompanhamento dos resultados do tratamento oferecido aos usuários, que permitam auxiliar nas mudanças estratégicas. O presente estudo objetivou avaliar a satisfação com serviço na perspectiva dos familiares cuidadores, considerando-os figura articuladora da relação usuário-serviço.

\section{Método}

O estudo foi aprovado pelo Comitê de Ética em Pesquisa, protocolo no 377/2008 - 2008.1.400.59.8, segundo os procedimentos básicos e éticos de respeito aos voluntários e à instituição.

\section{Participantes}

Participaram do presente estudo 54 familiares cuidadores de pessoas em situação de sofrimento mental que frequentavam serviço de internação parcial de uma cidade de médio porte do interior paulista. Os critérios de inclusão foram: a) o familiar ser o principal cuidador do usuário; b) o usuário estar frequentando o serviço de saúde mental há pelo menos um ano e c) o familiar residir no mesmo local que o usuário.

Para se constituir a amostra, foi realizado um levantamento de todos os prontuários dos usuários do serviço, identificando-se o total de 274. Destes, foram separados para consulta os prontuários dos usuários em cuidado semi-intensivo e intensivo, ou seja, usuários que, em função de seu quadro clínico, necessitam de maior frequência no acompanhamento pelo serviço, totalizando 108 . Verificou-se que, após o contato com os familiares dos usuários nos cuidados semi e intensivo, 16 usuários não estavam mais em acompanhamento no serviço; 23 possuíam condições em não-conformidade aos critérios estabelecidos e 15 recusaram-se a participar do estudo.
A amostra foi constituída por maioria do sexo feminino (62,96\%) e idade média igual a 52,98 anos. Dentre os participantes da pesquisa, $29,63 \%$ possuíam o ensino médio completo ou ensino superior incompleto, seguido de $27,78 \%$ com ensino fundamental completo. Em relação ao estado civil, possuíam companheiros e não possuíam companheiros $50 \%$ cada. Dos cuidadores participantes, a maioria possuía trabalho remunerado $(66,67 \%)$ e a renda familiar mais comum foi a de até um salário mínimo (27,78\%).

Como se pôde observar, 40,74\% dos cuidadores foram constituídos por mães e pais, sendo que as mães foram mais frequentemente identificadas como as principais cuidadoras (27,78\%). Em seguida, foram mais citados como cuidadores principais as irmãs $(14,81 \%)$, os pais $(12,96 \%)$ e os maridos (12,96\%).

Em relação aos usuários de serviço de saúde mental cujo familiar cuidador participou do presente estudo, predominou mulheres usuárias (55,56\%) e idade média de 44,89 anos. Em relação à escolaridade, $48,15 \%$ possuíam ensino médio completo ou ensino superior incompleto, seguido de $24,07 \%$ sem escolaridade, ou apenas ensino fundamental incompleto. Quanto ao estado civil, 75,93\% estavam sem companheiros, sendo que 29 nunca se casaram, nove eram separados/divorciados e três viúvos.

De acordo com registro médico no prontuário, a doença mais frequente dos usuários cujos cuidadores participaram do presente estudo foi esquizofrenia (46,30\%). Dentre os cuidadores, $68,52 \%$ sabiam o diagnóstico do usuário, tendo sido a esquizofrenia o diagnóstico mais frequentemente relatado pelos cuidadores. Em relação ao tempo de tratamento, foi observada média de 14,47 anos e de tempo de tratamento no serviço média de 8,45 anos.

\section{Instrumentos}

Para a avaliação da satisfação dos cuidadores, foi utilizada a Escala de Avaliação da Satisfação dos Familiares em Serviços de Saúde Mental (SATIS-Br), que foi reformulada a partir da escala original elaborada pela OMS, sendo então adaptada e validada para o contexto brasileiro (Bandeira et al., 2002). É composta por 62 itens, dentre os quais se incluem: a) questões quantitativas, que objetivam avaliar o grau de satisfação dos familiares em relação aos serviços; b) questões qualitativas e descritivas que se referem à opinião dos familiares sobre os serviços e c) dados sociodemográficos. A parte da escala que avalia o grau de satisfação dos familiares com o serviço de saúde mental inclui oito itens quantitativos, contendo respostas dispostas em escala ordinal do tipo Likert de cinco pontos. O questionário contém também 47 questões descritivas que visam obter informações complementares, tais como motivo da procura, condições físicas do serviço e acesso à instituição. Há, ainda, três questões qualitativas abertas, com o objetivo de obter informações mais detalhadas sobre as formas de atendimento e aspectos de maior e de menor satisfação. A escala apresentou consistência interna adequada, com alfa de Cronbach variando de 0,76 a 0,89 (Bandeira et al., 2002). 
Um questionário complementar foi também utilizado a fim de se efetuar um levantamento dos dados sociodemográficos dos cuidadores, bem como dos usuários cujos familiares participaram do estudo.

\section{Procedimentos de coleta e análise de dados}

A coleta foi realizada no domicílio do familiar, atendendose os preceitos éticos de privacidade. Os instrumentos foram aplicados pela pesquisadora, na forma de entrevista face a face. Inicialmente, foi ressaltado o caráter voluntário da colaboração e foram esclarecidas as condições de sua participação através do termo de consentimento livre e esclarecido, o qual foi lido e assinado pelos participantes. Os instrumentos foram aplicados segundo suas instruções particulares na seguinte ordem: Questionário sociodemográfico e SATIS-Br, sendo este posteriormente cotado segundo suas proposições técnicas. As questões abertas foram categorizadas segundo frequência de respostas. A coleta dos dados foi realizada no período de setembro de 2008 a julho de 2009 e a aplicação dos instrumentos foi realizada em tempo médio de duas horas. Quando não houve possibilidade de continuidade em um mesmo dia, nova visita era agendada a fim de finalizar a coleta, minimizando, assim, a interferência nos dados.

Os dados foram codificados para posterior tratamento estatístico. Para a definição do procedimento estatístico, foi aplicado, inicialmente, o Teste Kolmogorov-Smirnov. Devido à não-normalidade dos dados, foi efetuado o tratamento estatístico inferencial, através de estatística não-paramétrica, aplicando-se o Teste de Mann-Whitney e o Coeficiente de Correlação de Postos de Spearman, adotando-se valores de $\mathrm{p} £$ 0,05 . Os demais dados foram organizados através de estatística descritiva.

As questões abertas foram categorizadas segundo frequência de resposta, pautando-se nos princípios de Análise de Conteúdo proposta por Bardin (1979). Tal análise consiste num conjunto de técnicas, através de procedimentos de sistematização, para a descrição dos conteúdos das comunicações, a fim de se identificar núcleos de sentido a partir dos quais se pode realizar inferências e interpretações quanto aos seus significados.

Constitui-se por: a) pré-análise, leitura flutuante das comunicações, tomando-se contato exaustivo com o material e com seu conteúdo; b) exploração, que permite a codificação do material, feita através de recortes, agregação e enumeração, permitindo realizar uma representação do conteúdo capaz de esclarecer acerca das características do texto e c) tratamento dos resultados e interpretação, na qual se realizam inferências a partir da análise do material, constituindo a análise propriamente dita.

\section{Resultados}

A Tabela 1 apresenta a caracterização da satisfação dos cuidadores participantes com o serviço de saúde mental frequentado pelo familiar em sofrimento mental.
Tabela 1

Média, Desvio Padrão e Intervalo Mínimo-Máximo Obtido para os Fatores de Satisfação do Familiar com Serviço de Saúde Mental

\begin{tabular}{cccc}
\hline Fatores de Satisfação & Intervalo mín-máx & Média & $\begin{array}{c}\text { Desvio } \\
\text { Padrão }\end{array}$ \\
\hline Satisfação Global & $2,13-5,0$ & 4,00 & 0,66 \\
Resultados do tratamento & $1,33-5,0$ & 4,28 & 0,83 \\
Acolhida/Competência equipe & $1,33-5,0$ & 3,62 & 1,12 \\
Privacidade/Confidencialidade & $2,5-5,0$ & 3,98 & 0,59 \\
\hline
\end{tabular}

Verifica-se que o escore médio de satisfação global dos familiares em relação ao serviço frequentado pelo usuário foi de 4,00 . O fator que obteve escore médio mais elevado de satisfação se relaciona ao resultado do tratamento $(M=4,28)$ e o de menor satisfação, relacionado à satisfação com a acolhida e competência da equipe $(M=3,62)$. Uma vez que quanto mais próximo a cinco for o valor obtido maior a satisfação, pode-se dizer que os familiares cuidadores participantes apresentam uma satisfação de moderada a alta em relação aos aspectos avaliados.

$\mathrm{Na}$ Tabela 2, apresentam-se as porcentagens de respostas dos familiares às questões que avaliam as dimensões da satisfação com o serviço.

Tabela 2

Porcentagem de Respostas dos Familiares Cuidadores às Questões Referentes à Satisfação com Serviço (SATIS-Br) em Função da Percepção de Baixa ou Elevada Satisfação

\begin{tabular}{|c|c|c|c|c|}
\hline Fatores de Satisfação & $f$ & $\begin{array}{c}\text { Baixa } \\
\text { satisfação }\end{array}$ & $f$ & $\begin{array}{l}\text { Elevada } \\
\text { satisfação }\end{array}$ \\
\hline \multicolumn{5}{|l|}{ Resultados do tratamento } \\
\hline Serviço ajudou paciente & 2 & $3,70 \%$ & 47 & $87,03 \%$ \\
\hline Recebeu o cuidado necessário & 4 & $7,41 \%$ & 41 & $75,93 \%$ \\
\hline Usuário se beneficiou com o serviço & 1 & $1,85 \%$ & 49 & $90,74 \%$ \\
\hline \multicolumn{5}{|l|}{$\begin{array}{l}\text { Acolhida e competência da } \\
\text { equipe }\end{array}$} \\
\hline $\begin{array}{l}\text { Profissional compreendeu o proble- } \\
\text { ma do paciente }\end{array}$ & 0 & - & 33 & $61,11 \%$ \\
\hline $\begin{array}{l}\text { Equipe compreendeu o problema } \\
\text { do paciente }\end{array}$ & 0 & - & 39 & $72,22 \%$ \\
\hline Competência do terapeuta principal & 2 & $3,70 \%$ & 45 & $83,33 \%$ \\
\hline \multicolumn{5}{|l|}{$\begin{array}{l}\text { Privacidade e confidencialidade } \\
\text { do serviço }\end{array}$} \\
\hline $\begin{array}{l}\text { Medidas tomadas para assegurar } \\
\text { privacidade durante } 0 \text { tratamento }\end{array}$ & 1 & $1,85 \%$ & 48 & $88,89 \%$ \\
\hline $\begin{array}{l}\text { Medidas tomadas para assegurar } \\
\text { confidencialidade sobre } 0 \text { paciente }\end{array}$ & 2 & $3,70 \%$ & 47 & $87,03 \%$ \\
\hline
\end{tabular}

A Tabela 2 apresenta os dados descritivos relativos à satisfação com serviço avaliada através das respostas 1 e 2 (baixa satisfação) e 4 e 5 (elevada satisfação), em cada dimensão da SATIS-Br. Observa-se, pela tabela, maior frequência de elevada satisfação em todos os aspectos avaliados, principalmente entre itens relativos à dimensão "resultados do tratamento". 
Pela análise correlacional, verificou-se correlação negativa entre satisfação com os resultados do tratamento e a variável idade do usuário ( $p=0,006$ - Coeficiente de correlação de Spearman), indicando que à medida que aumenta a idade do usuário, menor é a satisfação do familiar com o tratamento. As demais análises não retornaram resultados estatisticamente significativos.

Quanto aos aspectos descritivos avaliados pelas demais questões do SATIS-Br, verificou-se que, dos familiares participantes, $70,37 \%$ referiram não terem participado no ano anterior de alguma atividade no serviço de saúde mental frequentado pelo usuário. Vale ressaltar que a questão citada enfoca o ano imediatamente anterior ao da coleta de dados efetuada. Dentre aqueles que relataram terem participado $(n=16), 12$ referiram a reunião de familiares como a atividade mais frequentemente acompanhada, seguido por quatro que referiram participação em atendimentos quando chamados no serviço. Grupo informativo, evento de fim de ano, participação no café da manhã e atividades artísticas também foram relatados. Do total de participantes, $57,40 \%$ nunca ou raramente acompanharam o usuário ao serviço.

Para chegar até o serviço, 33,33\% utilizavam condução própria e $29,63 \%$, transporte público. Dentre os cuidadores, $38,89 \%$ referiram ser mais ou menos fácil chegar até o local, sendo a localização moderadamente satisfatória.

Em relação ao contato com os profissionais, 42,60\% referiram nunca ou raramente terem estabelecido contato, seguido de $29,63 \%$ que referiram moderado contato. A acolhida da equipe foi mais frequentemente avaliada como amigável ou muito amigável (88,89\%).

Conforme apontaram $68,52 \%$ dos participantes, o profissional que atendeu ao usuário pela primeira vez o escutou bastante ou o tempo todo, parecendo que compreendeu o problema na visão de $61,11 \%$ dos cuidadores. Observou-se um número relevante de cuidadores que responderam que tais questões não se aplicavam, uma vez que não acompanharam os usuários no primeiro atendimento (37,04\%).

A maioria dos familiares considerou que o problema do usuário, quando foi admitido no serviço, dizia respeito a problema de saúde mental $(57,41 \%)$, sendo que para $27,78 \%$, tratava-se de um problema familiar. Para 59,26\% dos cuidadores, não havia outra opção de tratamento quando entraram em contato com o serviço. A qualidade do serviço foi avaliada como boa ou excelente por $88,89 \%$ dos cuidadores, estando satisfeitos ou muito satisfeitos com o serviço.

Para 70,37\% nunca houve dificuldade em obter informações da equipe do serviço. Entre os cuidadores que indicaram alguma dificuldade, verificou-se que estas se centraram na relação com a equipe ou com alguns membros da equipe, relacionando-se a: a) problemas com informações (informações escassas, falta de esclarecimento, não-consideração do conhecimento informal do cuidador) e b) dificuldades associadas ao atendimento ao familiar.

Dos respondentes, 79,63\% afirmaram terem recebido informações sobre a doença do usuário, sendo que, para $64,81 \%$, estas informações foram claras ou muito claras. Informações sobre o tratamento foram dadas para $79,62 \%$ dos cuidadores, sendo consideradas claras ou muito claras por $66,67 \%$. Dos cuidadores, $75,93 \%$ referiram não ter sido pedida sua opinião sobre o tratamento do usuário. Entre eles, $81,48 \%$ acreditavam que os serviços recebidos pelo usuário foram apropriados à sua necessidade. Dos participantes, $87,04 \%$ relataram nunca terem participado de decisão quanto ao planejamento e/ou realização de atividades, ou mesmo de avaliação das atividades no serviço. Por fim, $64,81 \%$ estavam satisfeitos com o conforto e aparência do serviço, sendo que $44,44 \%$ consideraram as condições das instalações boas e $38,89 \%$ as consideraram mais ou menos. Entre os participantes, $61,11 \%$ indicariam o serviço a alguém que estivesse precisando de ajuda semelhante, estando satisfeitos com relação aos serviços recebidos.

Em relação às questões abertas qualitativas presentes no questionário, os familiares cuidadores referiram como pontos positivos do serviço de saúde mental: a) equipe atenciosa, acolhedora e dedicada, oferecendo bom atendimento; b) atividades psicossociais destinadas ao usuário; c) resultados do cuidado/tratamento, com melhora do usuário; d) própria satisfação do usuário. Em contrapartida, relataram os seguintes aspectos negativos: a) aspectos da organização do serviço (rotatividade de pessoal, dificuldade com profissional específico; falta de confiança na equipe, demora pelo atendimento, poucos profissionais); b) proximidade com usuários com sintomatologia grave; c) aspectos físicos (condições físicas e limpeza); d) atividades escassas; e) excesso de usuários. A reunião de família foi avaliada tanto como aspecto de satisfação como de insatisfação, havendo cuidadores que gostam e cuidadores que não gostam dessas reuniões.

Quando questionados sobre sua percepção quanto à necessidade de aspectos a serem melhorados no serviço, 66,67\% dos participantes pontuaram sugestões que foram categorizadas e apresentadas na Figura 1, segundo ordem decrescente de ocorrência de resposta, como indicações para o aperfeiçoamento do serviço.

\begin{tabular}{|l|l|}
\hline A. Ampliação de atividades & $\begin{array}{l}\text { A.1. Destinadas aos usuários } \\
\text { A.2. Destinadas aos familiares }\end{array}$ \\
\hline \multirow{4}{*}{ B. Profissional } & $\begin{array}{l}\text { B.1. Aumento de pessoal } \\
\text { B.2. Formação continuada } \\
\text { B.3. Retorno de profissional } \\
\text { B.4. Remuneração }\end{array}$ \\
\hline \multirow{5}{*}{ C. Instalações físicas } & $\begin{array}{l}\text { C.1. Ampliação } \\
\text { C.2. Limpeza } \\
\text { C.3. Melhora } \\
\text { C.4. Aumento número de banheiros } \\
\text { C.5. Ampliação de área verde } \\
\text { C.6. Segurança }\end{array}$ \\
\hline D. Usuários & $\begin{array}{l}\text { D.1. Separação no serviço segundo grau de severidade } \\
\text { do diagnóstico } \\
\text { D.2. Atendimento na urgência / Internações rápidas } \\
\text { D.3. Controle de comparecimento ao serviço }\end{array}$ \\
\hline \multirow{3}{*}{ E. Família } & $\begin{array}{l}\text { E.1. Exigência da participação do familiar } \\
\text { E.2. Informações periódicas sobre doença / tratamento } \\
\text { do usuário }\end{array}$ \\
\hline
\end{tabular}

Figura 1: Quadro de fatores apontados como sugestão de melhora para o serviço de saúde mental 


\section{Discussão}

No que concerne à satisfação do cuidador com o serviço frequentado pelo usuário, pôde-se observar satisfação positiva em todas as dimensões avaliadas, a saber: resultados do tratamento, satisfação global, privacidade e confidencialidade do serviço e acolhida e competência da equipe, respectivamente. Bandeira et al. (2011) verificaram dados semelhantes. Destaca-se o valor positivo de tais avaliações, entretanto, ressalta-se que o excesso de respostas positivas por parte dos familiares vem sendo discutido na literatura, o que pode ser atenuado pelo uso de instrumentos multifatoriais (Bandeira et al., 2002), como o utilizado na presente pesquisa. A partir de dados complementares, pode-se ampliar a possibilidade de compreensão dos dados obtidos.

Observou-se relato mais frequente de não-participação em atividades voltadas ao familiar, assim como baixa incidência de acompanhamento do usuário em seu tratamento. Ainda, foi relatado pouco contato com os profissionais que integram a equipe do serviço, revelando distanciamento do familiar em relação ao tratamento do usuário.

Tais dados podem ser compreendidos dentro do contexto de tempo de tratamento do usuário, bem como considerando a visão dos participantes, tendo em vista sua participação no ano anterior. A satisfação pode estar atrelada como advindo da compreensão do serviço como oferecendo ao familiar libertação e alívio - enquanto o usuário é assistido no serviço, o familiar pode dar conta de outras demandas de sua vida, livre do encargo do cuidado que é transferido ao serviço. Mello e Furegato (2008) verificaram que os familiares consideram que o serviço propicia alívio da sobrecarga gerada pela convivência prolongada com o indivíduo com transtorno mental.

Além disso, o serviço de saúde mental consiste na maior fonte de ajuda ao familiar-cuidador, o que pode limitar as expressões de desagrado, além do que a coleta de dados foi realizada na forma de entrevista face a face, o que também pode levar a uma menor manifestação de insatisfação. Desta forma, a parceria família-serviço e a corresponsabilização, tão discutidas pelos paradigmas da reforma da assistência em saúde mental, não são efetivadas, ocorrendo, em contrapartida, a transferência e divisão de responsabilidades.

Neste sentido, pode-se apontar a sugestão de maior número de atividades destinadas aos usuários como sugestão de melhora do serviço. Pode-se supor que perceber o usuário ocupado, interessado e envolvido em algo, como num trabalho ou numa interação social, gere maior tranquilidade ao familiar tendo em vista a possibilidade de o usuário desfrutar de situações diversificadas e ampliar o convívio social, atendendo a expectativa do cuidador de que o usuário esteja bem, e ainda, libere o familiar para o atendimento a outras necessidades próprias, além de poder partilhar a responsabilidade pelo cuidado com outra instituição. Sugere-se que a indicação de que o serviço promova mais atividades aos usuários exista na tentativa de cumprir a função de propiciar ao cuidador alívio da tarefa do cuidado, possibilitando a saída do usuário de sua residência, assim como favorecer a percepção de maior engajamento social do usuário, questão esta afetada pela vivência do transtorno mental. No estudo de Kantorski et al. (2011) a questão da ampliação das atividades também foi apontada, como a criação de sala de esportes, ofertando-se mais atividades recreativas.

Indaga-se, no entanto, se caberia apenas ao serviço de saúde mental responder a esta demanda. Atividades desportivas, ocupacionais, de recreação, em articulação com cooperativas de trabalho, associações civis e comunitárias, que cumpram a função de reabilitação social, podem ser efetivas na redução da sobrecarga do cuidador e melhora da qualidade de vida do usuário. Segundo Fontes (2008), deve-se lidar com o sofrimento mental apoiando-se nas redes de sociabilidade presentes na comunidade e no cotidiano do usuário, fortalecendo seus vínculos.

Há, pois, que se falar em territorialidade, movimento de expansão dos serviços em direção à comunidade, rompendo as barreiras institucionais que delimitam e restringem os espaços para o cuidado. O serviço, como articulador da rede de cuidados, deve prover condições para a construção de novos espaços de promoção de saúde, aproximando serviço e família. Para tanto, recursos comunitários devem ser acionados, assim como a rede básica de saúde. Para Dimenstein, Galvão e Severo (2009), a ampliação da rede básica, por meio da Estratégia da Saúde da Família, pode favorecer a viabilização do atendimento comunitário. Espera-se, pois, uma maior inserção das equipes na comunidade local, numa abordagem multidisciplinar, e ações intersetoriais que integrem a assistência em saúde mental. Os recursos a serem acionados necessitam se situar próximo à localidade das famílias e do usuário, com acompanhamento longitudinal da atenção básica.

Os espaços destinados à família no serviço, tal como a reunião de família, mostraram-se pouco frequentados pelos participantes no ano anterior ao do estudo, assim como foi verificado baixo acompanhamento do tratamento do usuário no serviço pelo cuidador, o que permite discutir o papel atribuído ao familiar como principal cuidador, assim como o papel que o serviço de saúde mental cumpre para esses familiares.

A atribuição da condição de principal cuidador se deu considerando aquele familiar que fosse mais próximo e que mais estivesse associado ao tratamento do usuário. Apesar da baixa frequência do acompanhamento do serviço, pode-se supor que consistiram nos familiares mais envolvidos nas tarefas de cuidado e de supervisão, sendo aqueles com maiores adaptações do dia a dia, dada a função de cuidador desempenhada principalmente no lar.

Baixa participação do familiar no tratamento com pouco contato com o serviço foi constatada em outros estudos. Campos e Soares (2005) problematizaram a questão da adesão familiar, afirmando ser a sobrecarga emocional mais intensa entre cuidadores não-participantes. Ao mesmo tempo, assinalaram a falta de mecanismos dos serviços em propiciar um atendimento centrado na família que os auxilie no alívio da sobrecarga. Santos, Almeida, Venâncio e Delgado (2000), em um estudo visando a avaliação da inserção dos familiares em um serviço de saúde mental, verificaram pouca participação, 
embora seja incentivada a maior participação do familiar no tratamento. Maior participação familiar impacta direta e positivamente no tratamento do usuário, melhorando sua adesão e resposta ao tratamento (Bandeira \& Barroso, 2005) e vem sendo associada maior satisfação do familiar (Perreault et al, 2012).

Para Schrank e Olschowsky (2008), os serviços devem atuar na necessidade de inclusão do familiar nos serviços de saúde mental, visando construir mecanismos de suporte que facilitem a adesão e a integração da família. Para os autores, o convívio do usuário com a família, proposto pela reforma psiquiátrica, deve ser acompanhado de uma inserção na rede de saúde mental que promova a extensão e continuidade do cuidado. Segundo Pegoraro e Caldana (2006), as ações direcionadas à atenção ao familiar devem ir além do comparecimento em reuniões ou assembléias nos serviços de saúde.

Nesta perspectiva, vale discutir ações que efetivamente oportunizem a participação do familiar no tratamento do usuário, até mesmo porque há a sugestão de que se ampliem as atividades destinadas aos familiares, bem como de que estes possam contar com orientações e informações periodicamente, o que indica a necessidade de serem ainda mais incluídos no serviço. A disponibilização de atividades aos familiares nos serviços não garante em si a acessibilidade do cuidador. Tal questão envolve o oferecimento de condições que propiciem a atuação da família nos serviços. Como se verificou, a localização do serviço é considerada satisfatória para os familiares, sendo relativamente fácil chegar até o local. Verifica-se, no entanto, que a organização atual do serviço frequentado pelos usuários, cujos cuidadores participaram deste estudo, por vezes dificulta a real participação familiar. Como verificado no presente estudo, a maioria dos cuidadores afirmou exercer trabalho remunerado, o que pode confrontar com o horário comercial de funcionamento do serviço, momento em que atividades voltadas à família, tais como reuniões, são oferecidas. Além disso, em se tratando de um serviço público, atende pessoas com nível sócio-econômico que muitas vezes limita a oportunidade de locomoção até o serviço. Observa-se, pois, que pode haver certa incompatibilidade entre o que é oferecido e o que é possível para as famílias dos usuários. Horários flexíveis, funcionamento do serviço em horário integral, encontros comunitários que se situem próximo à residência dos familiares e dos usuários podem ser ações que facilitem e promovam a participação familiar.

Outra dificuldade que pode levar o familiar a não comparecer ao serviço pode se associar à dificuldade de lidar com o sofrimento mental. Nesse sentido, pode-se citar a referida necessidade que os familiares cuidadores apontaram de que, no serviço, haja separação entre os usuários segundo grau de severidade de sintomatologia. Tal indicação é sugestiva do impacto negativo do contato com o sofrimento mental e das dificuldades sentidas pelo familiar na função de cuidador. Em estudo qualitativo realizado por Ribeiro, Coimbra e Borges (2012) com grupos de familiares de usuários de CAPS, destacou-se a necessidade dos cuidadores quanto ao acolhimento e acompanhamento na relação e convivência com o usuário e o potencial de ajuda das ações grupais para o fortalecimento das famílias.

No presente estudo, maior satisfação esteve associada aos resultados do tratamento, assim como no estudo de Bandeira et al. (2011), sendo o aspecto de que o usuário se beneficiou com o cuidado recebido o de maior satisfação. Sugere-se que perceber que o tratamento mantém o usuário fora do surto pode contribuir para a satisfação do cuidador. No entanto, essa satisfação tende a diminuir conforme aumenta a idade do usuário. Pode-se supor que, ainda que haja certa acomodação e resignação frente à situação vivenciada, quanto mais velho o usuário maior a percepção do caráter crônico da doença mental e, consequentemente, maior insatisfação com os resultados do tratamento no sentido de percepção de baixa resolutividade. Na pesquisa de Rammohan, Rao e Subbakrishna (2002), idade mais avançada do usuário esteve associada a maior sobrecarga do cuidador. No entanto, o estudo de Bandeira e Barroso (2005) aponta a falta de clareza dessa relação, tendo o estudo de Bandeira et al. (2011) inclusive a relação inversa. Pode-se supor que tais diferenças devam se relacionar a características das amostras. Bandeira, Felicio e Cesari (2010) apontam a importância da avaliação quanto à percepção de mudança na vida do usuário em decorrência do tratamento, na perspectiva do familiar, devido a sua posição peculiar para identificar indicadores de melhora e piora do usuário, contribuindo, assim, para a readequação do serviço.

Para os participantes, foi predominante a percepção de que a dificuldade do usuário se deve a problema de saúde mental, sendo considerável, no entanto, a parcela de cuidadores que atribuíram a dificuldades familiares o sofrimento mental apresentado pelo usuário. Pode-se inferir que os familiares reconhecem a influência de fatores do convívio na problemática do usuário, cabendo estudar como se configuram e as formas de ajuda possíveis neste âmbito.

Os aspectos de menor satisfação se relacionaram à acolhida e competência da equipe, ainda que tenham sido valores moderados, assim como encontrado por Bandeira et al. (2011). Entre os aspectos avaliados, a percepção quanto à compreensão do profissional acerca do problema do usuário foi o que obteve menor satisfação. Neste sentido, pode-se apontar os aspectos positivos e negativos relatados pelos cuidadores. Se, por um lado, alguns cuidadores relataram poder contar com profissionais acolhedores, dispostos e atenciosos, também foi na relação com a equipe que perceberam as mais acentuadas dificuldades, seja no que diz respeito à dificuldade ao acesso e compartilhamento de informações, ou mesmo a aspectos do atendimento que demonstraram a desorganização do serviço e a falta de confiança na equipe.

Ainda, observa-se que os cuidadores apontaram, com frequência, sugestão de melhora do serviço associada aos profissionais da equipe, indicando ser a ampliação do quadro funcional, bem como investimentos na equipe necessários à adequada satisfação das necessidades dos usuários e de seus familiares. A tais investimentos encontra-se subjacente a concepção de que, ao serem satisfeitas as necessidades dos profissionais, amplia-se a possibilidade de um bom cuidado. 
Tais dados, em conjunto, indicam a importância da relação entre família e profissional/serviço para a satisfação com o tratamento, a qual necessita implementação e esforços dirigidos a uma maior participação e parceria mais efetiva, uma vez que a participação mais ativa como colaborador do tratamento e dos profissionais do serviço encontra-se fortemente associada ao grau de satisfação do familiar (Perreault et al., 2012). Aspectos do relacionamento entre família e equipe se encontraram como mais problemáticos, principalmente no que concerne à informação e à consideração do conhecimento informal dos cuidadores. Queixas quanto à falta de informações, à doença e ao tratamento, bem como orientações no cotidiano encontram-se entre as mais frequentes entre os familiares (Perreault et al., 2012).

Estudo de Ribeiro et al. (2012) aponta a aquisição de um conhecimento empírico por parte dos familiares cuidadores, construída no fazer do cuidado cotidiano a ser valorizado, com potencial de ajuda que pode ser compartilhado em ações grupais. Apesar da moderada satisfação com os aspectos de informação sobre doença e tratamento do usuário, aponta-se a necessidade de investimentos mais efetivos no profissional de saúde mental, instrumentalizando-o nas habilidades e competências de cuidado ao outro no que diz respeito à escuta, compreensão, informação, troca e efetiva inclusão do familiar na reabilitação do usuário.

$\mathrm{Na}$ visão da maioria dos participantes, não há, da parte do serviço, uma busca por inserir o familiar enquanto um dos atores no cuidado, solicitando-lhe opiniões, informações ou mesmo considerando sua participação nas decisões do projeto terapêutico ou das atividades no serviço destinadas ao usuário. Observa-se, no entanto, ações particulares segmentadas, que ocorrem no serviço ou no lar sem uma integração entre as diferentes instituições que assumem as ações de cuidado. Alguns cuidadores apontam inclusive a necessidade de que haja uma exigência quanto à participação do familiar no serviço e no tratamento do usuário, bem como ampliação das atividades ao familiar e encontros periódicos no intuito de fornecer informações sobre a evolução do usuário. Tais ações forçam a colaboração do familiar, exercendo um controle sobre sua participação. Questões associadas ao acolhimento da equipe não aparentam ser fonte de dificuldade, uma vez que a maioria dos participantes considera positivamente essa característica dos profissionais.

Ainda que os cuidadores tenham referido satisfação quanto às condições físicas do serviço, apontaram, com frequência, aspectos a serem melhorados nesse quesito, indicando a necessidade de reforma, ampliação e melhores condições das instalações. Pode-se supor que poder contar com o serviço já é de grande auxílio e satisfação, o que não indica que não sejam necessárias mudanças visando a um atendimento ainda melhor às necessidades dos usuários e de seus familiares.

\section{Considerações finais}

Os dados apontam a necessidade de que os serviços substitutivos possam, de acordo com a área de abrangência, localizar e otimizar recursos locais de ajuda e integração do usuário e, ainda, propiciar um acompanhamento longitudinal que envolva o conhecimento das condições de vida e a ajuda para lidar com questões de dificuldades do familiar. Para efetivar o encontro, não basta esperar pelo familiar, mas se deve ir até ele. É no contexto de vida comunitária, para além dos limites estabelecidos da saúde mental, que se deve ampliar, expandir a atenção psicossocial e promover a saúde e a qualidade de vida de todos os atores envolvidos no cuidado - profissionais, família e usuários -, atendendo as suas necessidades.

\section{Referências}

Almeida, P. F. (2002). O desafio da produção de indicadores para avaliação de serviços em saúde mental: um estudo de caso do centro de atenção psicossocial Rubens Côrrea/RJ (Dissertação de Mestrado, Escola Nacional de Saúde Pública da Fundação Oswaldo Cruz). Recuperado de http://www.arca. fiocruz.br/bitstream/icict/5043/2/468.pdf

Bandeira, M., \& Barroso, S. M. (2005). Sobrecarga das famílias de pacientes psiquiátricos. Jornal Brasileiro de Psiquiatria, 54(1), 34-46.

Bandeira, M., Felicio, C. M., \& Cesari, L. (2010). Percepção de mudanças pelos familiares como resultado do tratamento em serviços de saúde mental: Validação da escala EMP-familiares. Revista Brasileira de Psiquiatria, 32(3), 283-87.

Bandeira, M., Perreault, M., Mercier, C., Libério, M., \& Pitta, A. M. (2002). Escala de avaliação da satisfação dos familiares em serviços de saúde mental. Jornal Brasileiro de Psiquiatria, 51(3), 153-66.

Bandeira, M., Pitta, A. M. F., \& Mercier, C. (2000). Escala de avaliação da satisfação dos usuários em serviços de saúde mental: SATIS-BR. Jornal Brasileiro de Psiquiatria, 49(8), 293-300.

Bandeira, M., Silva, M. A., Camilo, C. A., \& Felicio, C. M. (2011). Satisfação de familiares em serviços de saúde mental. Jornal Brasileiro de Psiquiatria, 60(4), 284-93. Recuperado de http://www.scielo.br/pdf/jbpsiq/v60n4/ a09v60n4.pdf

Bardin, L. (1979). Análise de conteúdo. Lisboa: Edições 70

Campos, P. H. F., \& Soares, C. B. (2005). Representação da sobrecarga familiar e adesão aos serviços alternativos em saúde mental. Psicologia em Revista, 11(18), 219-37. Recuperado de http://www.pucminas.br/imagedb/ documento/DOC_DSC_NOME_ARQUI20060915161412.pdf

Costa, C. S., Bandeira, M., Cavalcanti, R. L. A., \& Scalon, J. D. (2011). A percepção de pacientes e familiares sobre os resultados do tratamento em serviços de saúde mental. Cadernos de Saúde Pública, 27(5), 995-1007. doi: 10.1590/S0102-311X2011000500017

Dimenstein, M., Galvão, V. M., \& Severo, A. K. S. (2009). O apoio matricial na perspectiva de coordenadoras de equipes de saúde da família. Pesquisas e Práticas Psicossociais, 4(1), 37-48.

Donabedian, A. (1988). The assessment of technology and quality. A comparative study of certainties and ambiguities. International Journal of Technology Assessment in Health Care, 4(4), 487-496.

Duarte, M. L. D. (2007). Avaliação da atenção aos familiares num centro de atenção psicossocial: Uma abordagem qualitativa (Dissertação de Mestrado não publicada. Escola de Enfermagem de Ribeirão Preto, Ribeirão Preto).

Esperidião, M. A., \& Trad, L. A. B. (2005). Avaliação de satisfação de usuários. Ciência e Saúde Coletiva, 10 (Supl.), 303-12. doi: 10.1590/S141381232005000500031

Fontes, B. A. S. M. (2008). Dos pavilhões às ruas: A âncora territorial da reforma psiquiátrica. Revista de Terapia Ocupacional da Universidade de São Paulo, 19(3), 183-92. doi: 10.11606/issn.2238-6149.v19i3p183-192

Kantorski, L. P., Coimbra, V. C. C., Silva, E. N. F., Guedes, A. C., Cortes, J. M., \& Santos, F. (2011). Avaliação qualitativa de ambiência num Centro de Atenção Psicossocial. Ciência \& Saúde Coletiva, 16(4), 2059-2066. doi: 10.1590/S1413-81232011000400005 
Kantorski, L. P., Jardim, V. M. R., Wetzel, C., Olschowsky, A., Schenider, J. F., R. F., Heck, R. M., ... \& Sousa, A. S. (2009). Contribuições do estudo de avaliação dos centros de atenção psicossocial da Região Sul do Brasil. Cadernos Brasileiros de Saúde Mental, 1(1), 1-9. Recuperado de http:// incubadora.periodicos.ufsc.br/index.php/cbsm/article/view/1015/1140

Kantorski, L. P., Machado, R. A., Lemões, M. A. M., Quadros, L. C. M., Coimbra, V. C. C., \& Jardim, V. M. R. (2012). Avaliação da estrutura e processo na visão dos familiares de usuários de saúde mental. Ciência, Cuidado e Saúde, 11(1), 173-180. doi: 10.4025/cienccuidsaude.v11i1.18875

Mello, R., \& Furegato, A. R. F. (2008). Representações de usuários, familiares e profissionais acerca de um Centro de Atenção Psicossocial. Revista de Enfermagem, 12(3), 457-64. doi: 10.1590/S1414-81452008000300010

Moreno, V., \& Alencastre, M. B. (2004). A família do portador de sofrimento psíquico e os serviços de saúde mental: Estudo de caso. Acta Scientiarum Health Sciences, 26(1), 175-81. doi: 10.4025/actascihealthsci.v26i1.1653

Organização Mundial da Saúde. (2001). Relatório sobre a Saúde no Mundo 2001. Saúde mental: nova concepção, nova esperança. Geneva (SW): OMS.

Pegoraro, R. F, \& Caldana, R. H. L. (2006). Sobrecarga de familiares de usuários de um centro de atenção psicossocial. Psicologia em Estudo, 11(3), 569-77.

Pereira, M. A. O., \& Cais, D. P. (2001). A percepção de familiares de pacientes psiquiátricos a respeito do serviço de saúde oferecido. Revista Gaúcha de Enfermagem, 22(2), 90-101. Recuperado de http://seer.ufrgs.br/index. $\mathrm{php} /$ RevistaGauchadeEnfermagem/article/view/4381

Perreault, M., Rousseau, M., Provencher, H., Roberts, S., \& Milton, D. (2012). Predictors of caregivers' satisfaction in mental health services. Community Mental Health Journal, 48(2), 232-237.
Pitta, A. M. F. (1996). Qualidade de serviços de saúde mental: Desafios para a epidemiologia. Jornal Brasileiro de Psiquiatria, 45(6), 313-321.

Rammohan, A., Rao, K., \& Subbakrishna, D. K. (2002). Religious coping and psychological wellbeing in careers of relatives with schizophrenia. Acta Psychiatrica Scandinavic, 105, 356-362.

Ribeiro, J. P., Coimbra, V. C. C., \& Borges, A. M. (2012). Grupos de familiares de um centro de atenção psicossocial: Experiências de seus usuários. Revista de Enfermagem da UFSM, 2(2), 375-385. Recuperado de http://cascavel. ufsm.br/revistas/ojs-2.2.2/index.php/reufsm/article/view/4582

Santos, N. S., Almeida, P. F.,Venâncio, A. T., \& Delgado, P. G. (2002). A autonomia do sujeito psicótico no contexto da reforma psiquiátrica brasileira. Psicologia: Ciência e Profissão, 20(4), 46-53. doi: 10.1590/S141498932000000400006

Schneider, J. F., Camatta, M. W., Nasi, C., Adamoli, A. N., \& Kantorski, L. P. (2009). Avaliação de um centro de atenção psicossocial brasileiro. Ciencia y Enfermaría, 25(3), 91-100. Recupeado de http://hdl.handle. net/10183/49801

Schrank, G., \& Olschowsky A. (2008). Centro de atenção psicossocial e as estratégias para inserção da família. Revista da Escola de Enfermagem, 42(1), 127-134. Recuperado de http://hdl.handle.net/10183/69671

Vasconcelos, E. M. Avaliação de serviços no contexto da desinstitucionalização psiquiátrica: revisão de metodologias e estratégias de pesquisa. Jornal Brasileiro de Psiquiatria, 44(4), 189-197.

Ana Flávia de Oliveira Santos, Mestre em Psicologia pela Faculdade de Filosofia, Ciências e Letras de Ribeirão Preto - USP, especialista em Psicologia Clínica pelo Conselho Federal de Psicologia. Endereço para correspondência: Departamento de Psicologia - Faculdade de Filosofia, Ciências e Letras de Ribeirão Preto - Universidade de São Paulo Av. dos Bandeirantes, 3900 - CEP - 14040901, Ribeirão Preto - SP Brasil. Telefone: 16-36023660 FAX: 16-3602-4835. E-mail: anafolisan@yahoo.com.br

Cármen Lúcia Cardoso, Professora Associada do Departamento de Psicologia da Faculdade de Filosofia, Ciências e Letras de Ribeirão Preto - USP (MS4), Docente do curso de Pós-Graduação em Psicologia (USP. E-mail: carmen@ffclrp.usp.br 